\title{
Consumo de alcohol en mujeres universitarias del Sur del Estado de Veracruz,
} México*

\author{
Alcohol Consumption in University Female Students from the South of the State of Veracruz, Mexico \\ Consumo de álcool em mulheres universitárias do Sul do Estado de Veracruz, México
}

\author{
Leticia Cortaza Ramirez ${ }^{\mathrm{a}}$ \\ Universidad Veracruzana, México \\ leticortaza@hotmail.com \\ ORCID: https://orcid.org/0000-0002-0592-7695 \\ Francisca Elvira Blanco Enríquez \\ Universidad Veracruzana, México \\ ORCID: https://orcid.org/0000-0001-6802-7486
}

DOI: https://doi.org/10.11144/Javeriana.ie22.camu

Recibido: 18 Mayo 2020

Aceptado: 22 Mayo 2020

Publicado: 30 Diciembre 2020

\section{Resumen:}

Introducción: el consumo de alcohol en las mujeres es una problemática cada día mayor, debido al aumento en los patrones nocivos y la edad a la que inician el consumo, por lo que representa un tema de vital relevancia en la agenda de la salud pública mundial. Objetivo: identificar el consumo de alcohol en mujeres universitarias del Sur del Estado de Veracruz, México. Método: estudio cuantitativo, descriptivo y transversal. Participaron 229 mujeres estudiantes de una universidad tecnológica de la zona sur del estado de Veracruz México, seleccionadas mediante muestreo probabilístico aleatorio simple, estratificado. Se utilizó un instrumento de recolección de datos personales y el Test de Identificación de los Trastornos debidos al Consumo de Alcohol (Audit). Resultados: se identificó que la media de edad de inicio de consumo fue de 17,1 años, 55,8\% de las participantes refirieron consumo de alcohol en el último año, la bebida preferida fue la cerveza (27\%), 67,2\% de las universitarias tiene consumo de bajo riesgo, 27,3\% consumo de riesgo, 4,7\% consumo perjudicial y 0,8\% consumo dependiente. Las estudiantes de Ingeniería Química Industrial reportan mayor consumo de riesgo $(11,7 \%)$, perjudicial $(1,6 \%)$ y dependiente $(0,8 \%)$. Conclusiones: más de la mitad de las participantes reportaron ingerir bebidas alcohólicas, con patrones de consumo de riesgo, mostrando la vulnerabilidad en la que se encuentran para adoptar conductas que ponen en riesgo su salud.

Palabras clave: Consumo de bebidas alcohólicas, consumo de alcohol en la universidad, estudiantes, bebidas alcohólicas, conducta de riesgo.

\section{Abstract:}

Introduction: the consumption of alcohol in women is a problem that grows every day due to the increase in harmful patterns and the age at which they start consumption. Therefore, it has become an issue of utmost relevance in the global public health agenda. Objective: to identify alcohol consumption in university female students from the south of the State of Veracruz, Mexico. Method: quantitative, descriptive, and cross-sectional study in which 229 female students from a technological university in the southern zone of the state of Veracruz, Mexico, participated. They were selected by simple, stratified random probability sampling. A personal data collection instrument and the Alcohol Use Disorders Identification Test (AUDIT) were used. Results: we identified that the mean age when consumption started was 17.1 years; $55.8 \%$ of the participants reported alcohol consumption in the last year; the preferred drink was beer (27\%); 67.2\% of the university students had low risk consumption, 27.3\% had high-risk consumption, 4.7\% harmful consumption, and $0.8 \%$ dependence consumption. Industrial Chemistry Engineering students report a higher highrisk (11.7\%), harmful (1.6\%), and dependence (0.8\%) consumption. Conclusions: more than half of the participants reported drinking alcoholic beverages, with high-risk consumption patterns, showing how vulnerable they are to adopt behaviors that put their health at risk.

Keywords: Consumption of alcoholic beverages, alcohol consumption at the university, students, alcoholic beverages, high-risk behavior.

\section{Resumo:}

Introdução: o consumo de álcool em mulheres é uma problemática a cada dia maior, devido ao aumento dos padrões nocivos e à idade em que iniciam o consumo, pelo qual representa um tema de vital relevância na agenda global de saúde pública. Objetivo:

Notas de autor

a Autora de correspondencia. Correo electrónico: leticortaza@hotmail.com 
identificar o consumo de álcool em mulheres universitárias do Sul do Estado de Veracruz, México. Método: estudo quantitativo, descritivo e transversal. Participaram 229 mulheres alunas de uma universidade tecnológica da zona sul do estado de Veracruz México, selecionadas mediante amostragem probabilística aleatória simples, estratificada. Foi utilizado um instrumento de coleta de dados pessoais e o Teste de Identificação de Transtornos devidos ao Consumo de Álcool (Audit). Resultados: identificou-se que a idade média de início de consumo foi 17,1 anos, 55,8\% das participantes referiram consumo de álcool no último ano, a bebida preferida foi cerveja (27\%), 67,2\% das universitárias tem consumo de baixo risco, 27,3\% consumo de risco, 4,7\% consumo prejudicial e 0,8\% consumo dependente. As alunas de Engenharia Química Industrial relatam maior consumo de risco (11,7\%), prejudicial $(1,6 \%)$ e dependente $(0,8 \%)$. Conclusões: mais da metade das participantes relatam ingerir bebidas alcoólicas, com padrões de consumo de risco, evidenciando a vulnerabilidade em que estão para adotar comportamentos que colocam em risco sua saúde.

Palavras-chave: Consumo de bebidas alcoólicas, consumo de álcool na universidade, alunos, bebidas alcoólicas, conduta de risco.

\section{Introducción}

El consumo de alcohol es en un problema de salud pública, que afecta a personas y sociedades de diferentes maneras; específicamente en las mujeres, reportes de la Organización Panamericana de la Salud (OPS) (1) indican que el número de mujeres que beben actualmente ha aumentado en el mundo; destacan que los principales factores que contribuyeron a la carga de mortalidad y los Años de Vida Ajustados por Discapacidad (AVAD) atribuibles al alcohol en esta población, fueron las enfermedades cardiovasculares, enfermedades digestivas y los traumatismos: a nivel mundial, se calcula que 46 millones de mujeres padecen trastornos por consumo de alcohol.

En la mujer, la ingesta de alcohol aun en pequeñas cantidades se ha encontrado asociado a diversas enfermedades, como el cáncer de mama, enfermedades cardíacas, lesiones por traumatismo, depresión, dependencia al alcohol y cirrosis hepática entre otras. La evidencia muestra que las mujeres son más vulnerables a los efectos nocivos del alcohol $(2,3)$.

El incremento en el consumo de las mujeres se observa en diversos países (4), sin embargo, la OPS (5) destaca que el consumo nocivo de alcohol es más elevado en la Región de las Américas, donde 47\% de hombres y 20,3\% de mujeres consumidoras (mayores de 15 años), informaron haber tenido consumo excesivo episódico de alcohol; señala además que cada 83 segundos se pierde una vida con causas relacionadas al consumo de alcohol.

Diversos estudios ponen de manifiesto el problema que representa actualmente el aumento del consumo de alcohol en las mujeres; como el realizado en Chile por el Servicio Nacional para la Prevención y Rehabilitación del Consumo de Drogas y Alcohol, donde 42,5\% de mujeres y 55,3\% de hombres afirmaron consumir alcohol; cifras que hace 20 años eran de $30 \%$ y $50 \%$ respectivamente, observando que la tendencia en el patrón de consumo ha cambiado, con un aumento considerable en las mujeres (6).

En Colombia, una investigación realizada en universitarios, indica que las mujeres reportaron un porcentaje cercano al de los hombres en el consumo problemático de alcohol (7). De igual forma, en Brasil un estudio en universitarios de Enfermería encontró que el consumo de alto riesgo predominó en las mujeres $(64,5 \%)$ con una marcada diferencia con los hombres (35,5\%) (8).

En México, el problema tienen un comportamiento similar y se muestra en los resultados de la última Encuesta Nacional de Consumo de Drogas, Alcohol y Tabaco (Encodat) 2016-2017, donde se comparan los resultados del año 2011 y 2016, observando un aumento preocupante en el consumo en el último mes en mujeres de 12 a 17 años, el cual pasó de 11,6\% a 15,4\%; el consumo excesivo durante el último año se incrementó más de 3 veces, pasó de 2,2\% en 2011 a 7,7\% en 2016; a diferencia de los hombres en quienes no se encontró variaciones significativas (9).

$\mathrm{Al}$ respecto, la Encuesta Nacional de Salud y Nutrición (Ensanut) 2018 (10) reporta que, en la población mexicana mayores de 20 años, el consumo de alcohol en el último año fue de 49,9\% en mujeres y $80.6 \%$ 
hombres; aunque en la frecuencia de consumo ocasional las mujeres refieren mayor consumo $(75,9 \%)$ que los hombres (47\%).

Así mismo, un estudio realizado en jóvenes universitarios de Monterrey Nuevo León, México (11), encontró diferencias significativas en el tipo de consumo por sexo $\left(\chi^{2}=11,07, \mathrm{p}<0,001\right)$, indicaron que el consumo de bajo riesgo $(30,3 \%)$ y de riesgo fue mayor en las mujeres $(33,3 \%)$ que en los varones. Otro estudio realizado en universitarios mexicanos del estado de Puebla reporta diferencias significativas en las proporciones del consumo de alcohol sensato $(\chi 2=4,567, \mathrm{p}=0,033)$ y dañino $(\chi 2=11,935, \mathrm{p}=0,001)$, con mayor proporción en las mujeres; en el consumo dependiente no se presentó diferencia significativa $(\chi 2=$ $0,028, \mathrm{p}=0,866)(12)$.

Lo anterior pone en evidencia el aumento del consumo de alcohol en la mujer, que en algunos casos supera al consumo realizado por los hombres, aunado a esta conducta de riesgo, puede agregarse la vulnerabilidad física de las mujeres a los efectos nocivos del alcohol, particularmente la actividad de la enzima alcoholdeshidrogenasa gástrica la cual interfiere en el metabolismo del alcohol, y que es menor en las mujeres que en los hombres, lo que hace que ellas con una misma cantidad de alcohol ingerida tengan una mayor concentración de alcohol en sangre que los hombres (13), y lleguen a la embriaguez más rápidamente, llevándolas a realizar conductas de riesgos como el sexo sin protección.

Estudios señalan, que existen diversos factores que pueden incentivar el consumo de bebidas alcohólicas en las mujeres; como los factores socioeconómicos, culturales, amigos consumidores y el ambiente universitario entre otros $(5,13)$.

La etapa universitaria, se sabe que cobra especial relevancia entre los jóvenes, ya que es un espacio de retos académicos, pero también de retos sociales y búsqueda de nuevas sensaciones y es en este periodo, donde las reuniones y convivencia son importantes por la búsqueda de aceptación a los grupos de iguales; y se convierte en un espacio de convivencia, don el alcohol está al alcance y disponibilidad, como una forma de crear un vínculo de pertenencia y sociabilidad, lo que puede llevar a las estudiantes a iniciar o aumentar el consumo de alcohol.

En lo antes descrito, se puede observar como el consumo de alcohol en la mujer se incrementa cada día más, como su propia naturaleza la hace más vulnerable y como el ambiente en el que se inserta al llegar a la vida universitaria la coloca en riesgo de iniciar o aumentar el consumo. De ahí la importancia de identificar el consumo de alcohol en estas jóvenes, lo que permitirá describir y entender la situación que viven actualmente las universitarias. Los resultados obtenidos pueden ser un eje fundamental en la búsqueda de estrategias de prevención adecuadas, las cuales pueden ser guiadas por enfermería, como pieza clave en el diseño e implementación de programas de salud, así mismo representa una oportunidad para realizar intervenciones dirigidas a la prevención del consumo de alcohol en este grupo poblacional. Por esta razón, la presente investigación buscó identificar el consumo de alcohol en mujeres universitarias del Sur del Estado de Veracruz, México.

\section{Método}

El estudio fue de tipo descriptivo y transversal; la población fue de 563 mujeres estudiantes de una universidad tecnológica de la Zona Sur del Estado de Veracruz, México. Las participantes se seleccionaron mediante un muestreo probabilístico aleatorio simple estratificado. La muestra final quedo integrada por 229 universitarias, seleccionadas de acuerdo con el tipo de carrera que se encontraban cursando. Se incluyeron a las estudiantes inscritas en el período febrero-mayo de 2018 y que aceptaran participar de forma voluntaria en la investigación.

Para la obtención de la información se utilizó un instrumento de recolección de datos personales que indagó la edad, el inicio del consumo de bebidas alcohólicas, los días de preferencia y el motivo para el consumo, así 
como la ingesta en el último año, mes y la semana previa al estudio, se utilizó además el Test de Identificación de Trastornos Relacionados con el Uso del Alcohol (Audit), integrado por 10 ítems, las puntuaciones oscilan de 0-7 puntos para el consumo sin riesgo, de 8 a 15 consumo de riesgo, 16 y 19 es consumo perjudicial y puntuaciones iguales o mayores a 20 identifican consumo dependiente. El cuestionario es un instrumento con validez transcultural que ha sido probado en población mexicana con una sensibilidad del 90\%, una especificidad del $94 \%$ y una confiabilidad aceptable $(0,80$ a 0,89$)$ (14). En este estudio el cuestionario obtuvo un Alpha de Cronbach de 0,85.

En el aspecto ético, la investigación se apegó a lo señalado en las disposiciones de la Ley General de Salud Mexicana en Materia de Investigación para la Salud, en el Titulo Segundo Capítulo I, artículos 13, 14, 16 y 21 (15), donde se estipula que deberá prevalecer el respeto a la dignidad y bienestar del ser humano sujeto de estudio, contar con el consentimiento informado por escrito y proteger la privacidad el individuo mediante el anonimato. Se respetaron de igual forma las disposiciones internacionales sobre buena práctica en investigaciones en seres humanos, apegándose a la Declaración de Helsinki. El proyecto contó además con la aprobación del comité de Investigación y Ética de la Facultad de Enfermería Campus Minatitlán de la Universidad Veracruzana. Una vez autorizado el proyecto, se solicitó el permiso a los directivos de la institución educativa donde se realizó el estudio, otorgado el permiso, se solicitó la lista de las estudiantes de acuerdo a la carrera que estudiaban y se les invito a participar en el estudio. Antes de la aplicación de los instrumentos se les solicitó a las participantes la firma del consentimiento informado y se les garantizó el anonimato en todo momento. La aplicación del instrumento se realizó en un tiempo aproximado de 20 minutos, y se llevó a cabo en un espacio destinado por la institución educativa. Los datos recabados, fueron procesados en el paquete estadístico Statistical Package for the Social Sciences (SPSS) versión 20. Para el análisis se utilizó la estadística descriptiva, se calcularon las frecuencias, porcentajes y medidas de tendencia central como la media y mediana.

\section{Resultados}

Participaron en el estudio 229 estudiantes entre 18 y 35 años, con una media de 20,76 2344 . El inicio de consumo de bebidas alcohólicas se dio entre los 12 y 23 años, con una media de 17,17 \pm 2170 . En lo referente al consumo de alcohol, 55.9\% de las participantes respondió que si consumieron e indicaron como bebidas de preferencia la Cerveza (27\%) y el Whisky (14,8). Mencionaron que los días preferidos para realizar el consumo fueron el viernes $(14,4 \%)$ y sábado $(42,4 \%)$. Revelaron además como motivos principales del consumo, la convivencia con amigos $(21 \%)$ y divertirse $(17,8 \%), 84,3 \%$ señala consumo en su círculo de amigos.

En lo referente al consumo de bebidas alcohólicas, la Figura 1, muestra que la mayoría de las estudiantes $(59,8 \%)$ refiere haber realizado la ingesta de bebidas alcohólicas en el último año, $45,4 \%$ reporta haber consumido en el último mes y 22,7\% mantuvo el consumo en la última semana previa al estudio. 


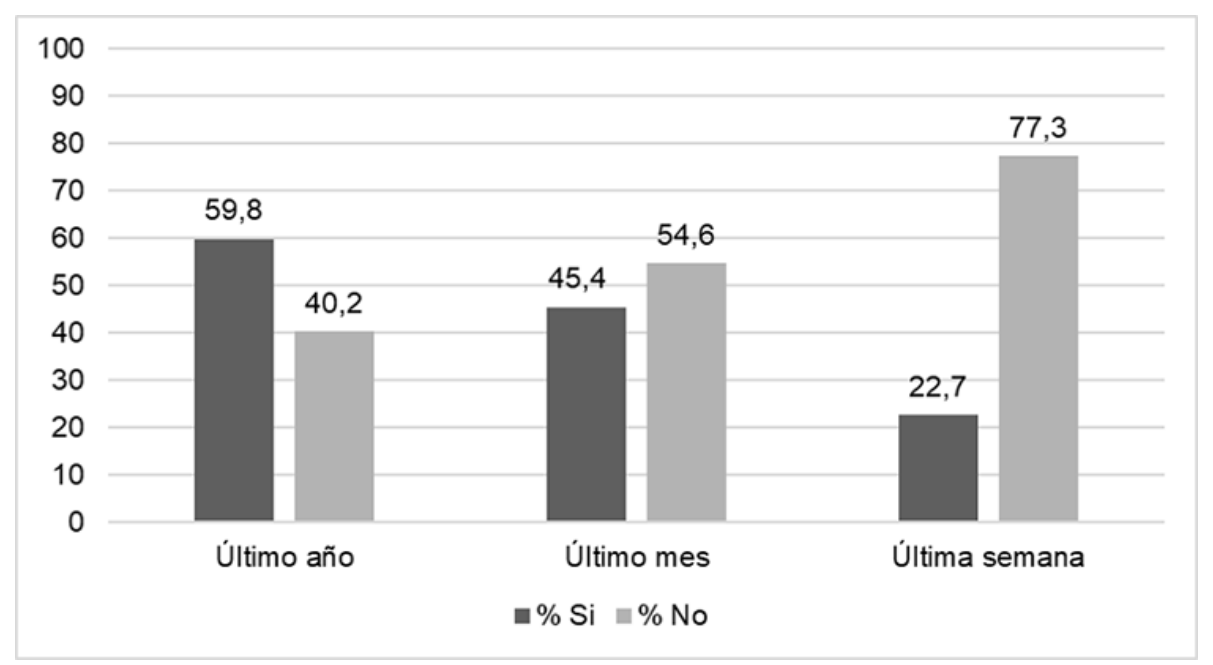

FIGURA 1.

Valores porcentuales del consumo de alcohol en estudiantes universitarias Fuente: elaboración propia

En lo que respecta a los patrones de consumo de alcohol (Tabla 1), predominó el consumo de bajo riesgo $(67,2 \%)$, sin embargo, un porcentaje considerable (27,3\%) de las jóvenes universitarias refiere consumo de riesgo; es importante destacar que existe consumo perjudicial $(4,7 \%)$ y dependiente $(0,8 \%)$ de acuerdo a lo señalado por el Audit. Este resultado pone en evidencia el consumo problemático de algunas estudiantes, así como la necesidad de realizar actividades de prevención, para que los niveles de riesgo no aumenten.

TABLA 1.

Consumo de alcohol en estudiantes clasificadas de acuerdo con el Test de Identificación de Trastornos Relacionados con el Uso del Alcohol (Audit)

\begin{tabular}{lcc}
\hline \multicolumn{1}{c}{ Tipo de consumo } & $\boldsymbol{f}$ & $\mathbf{\%}$ \\
\hline Bajo riesgo & 86 & 67,2 \\
De riesgo & 35 & 27,3 \\
Perjudicial & 6 & 4,7 \\
Dependiente & 1 & 0,8 \\
\hline
\end{tabular}

Fuente: elaboración propia.

Cuando se analizó el consumo de acuerdo con la carrera que estudiaban, se encontró que las jóvenes que estudian ingeniería química industrial reportan mayor consumo de riesgo (11,7\%), perjudicial (1,6\%) y dependiente (0,8\%); así mismo las estudiantes del área de mantenimiento industrial tuvieron un comportamiento similar en el consumo de riesgo $(9,4 \%)$ y perjudicial $(1,6 \%)$ (Tabla 2$)$. Es importante destacar que tanto la ingeniería química industrial, como mantenimiento industrial son carreras donde predominan los hombres, situación que puede llevarlas a incrementar el consumo por la convivencia que tienen con sus compañeros varones, si se toma en cuenta que más del $80 \%$ de las participantes tiene amigos consumidores, y además refieren realizar el consumo por convivencia y diversión. 
TABLA 2.

Consumo de alcohol por carrera que estudian las universitarias

\begin{tabular}{|c|c|c|c|c|c|c|c|c|}
\hline \multirow{3}{*}{$\begin{array}{l}\text { Carrea que } \\
\text { estudian }\end{array}$} & \multicolumn{8}{|c|}{ Patrón de Consumo } \\
\hline & \multicolumn{2}{|c|}{ Consumo de bajo riesgo } & \multicolumn{2}{|c|}{ Consumo de riesgo } & \multicolumn{2}{|c|}{ Consumo perjudicical } & \multicolumn{2}{|c|}{ Consumo dependiente } \\
\hline & $f$ & $\%$ & $f$ & $\%$ & $f$ & $\%$ & $f$ & $\%$ \\
\hline $\begin{array}{l}\text { Administación de } \\
\text { Recursos }\end{array}$ & 1 & 0.8 & 0 & 0 & 0 & 0 & 0 & 0 \\
\hline $\begin{array}{l}\text { Conmanos } \\
\text { Contaduia }\end{array}$ & 3 & 2,3 & 6 & 4,7 & 1 & 0,8 & 0 & 0 \\
\hline Mecaróninica & 1 & 0,8 & 2 & 1,6 & 1 & 0,8 & 0 & 0 \\
\hline $\begin{array}{l}\text { Mantenimiento } \\
\text { Industrial }\end{array}$ & 17 & 13,3 & 12 & 9,4 & 2 & 1,6 & 0 & 0 \\
\hline Quimica Industrial & 47 & 36.7 & 15 & 11,7 & 2 & 1,6 & 1 & 0,8 \\
\hline $\begin{array}{l}\text { Tecenologias de la } \\
\text { Informacion }\end{array}$ & 17 & 13,3 & 0 & 0 & 0 & 0 & 0 & 0 \\
\hline
\end{tabular}

Fuente: elaboración propia.

\section{Discusión}

El presente estudio permitió identificar el consumo de alcohol en mujeres estudiantes universitarias de una institución pública del sur del estado de Veracruz en México. Los hallazgos evidenciaron la existencia del consumo de alcohol en la mayoría de las mujeres que participaron en el estudio, con edad promedio de inicio en el consumo de 17,1 años, edad similar a la reportada por la Encodat 2016-2017 (9), quien indica como edad de inicio del consumo 17.9 años; sin embargo, este mismo resultado difiere de los hallazgos de García (4), Díaz (8) y Telumbre-Terrero (16) quienes muestran en sus investigaciones que la edad para el inicio del consumo de alcohol se da a los 15, 23 y 16 años, respectivamente.

En lo que respecta a la bebida de preferencia, las jóvenes mencionan que la bebida preferida fue la cerveza (27,1\%), resultado similar a lo reportado por la Encodat 2016-2017 (9) y Gómez y colaboradores (17), que revelan en sus hallazgos que la cerveza también fue la bebida de preferencia por la mayor parte de los encuestados.

La convivencia con los amigos $(21,8 \%)$ fue señalada como el principal motivo que tienen las jóvenes para realizar el consumo de bebidas alcohólicas, hallazgo similar al de Gómez y colaboradores (17) quienes indican en sus resultados en jóvenes universitarios de Guadalajara que la mayoría prefiere ingerir bebidas alcohólicas con amigos $(60,4 \%)$.

$\mathrm{Al}$ respecto, un estudio realizado desde el abordaje cualitativo menciona que el consumo de alcohol constituye un dispositivo de sociabilización, de apertura a compartir con otros, respondiendo a una necesidad social y simbólica de los sujetos (18).

Sobre el consumo en el último año, el 59,8\% de las jóvenes refirió haber consumido bebidas alcohólicas en este período de tiempo, dato superior a lo reportado en la Encodat 2016-2017 (9) y la Encuesta Nacional de Salud y Nutrición (Ensanut) 2018 (10), quienes señalaron señalan que 27,2\% y 49,9\%, respectivamente, consumió alcohol en el último año.

En cuanto a los patrones de consumo, se encontró predominio del patrón de bajo riesgo $(67,2 \%)$, seguido por el de riesgo (27,3\%), perjudicial (4,7\%) y dependencia (0,8\%), comportamiento similar al estudio de Alonso y colaboradores (11) en jóvenes universitarios de Monterrey, Nuevo León, México, que encontró diferencias significativas para el tipo de consumo por sexo $\left(\chi^{2}=11,07, \mathrm{p}<0,001\right)$, con mayor proporción del consumo de bajo riesgo (30,3\%) y el de riesgo en el sexo femenino (33,3\%).

Cuando se analiza el patrón de consumo de acuerdo a la carrera que estudian, los hallazgos indican que las jóvenes que estudian ingeniería química industrial $(11,7 \%)$ y mantenimiento industrial $(9,4 \%)$ son las que reportaron mayor consumo de riesgo, es importante señalar que en ambas carreras predominan los hombres, por lo que ellas pueden estar más expuestas a la presión social para el consumo ejercida por el grupo de amigos o compañeros de estudio, así como por la accesibilidad a las bebidas en los entornos universitarios, o por las normas sociales percibidas por las estudiantes, que las llevan a incrementar su consumo de forma riesgosa. 
Los hallazgos del estudio aportan conocimiento a la enfermería, principalmente para el primer nivel de atención, campo de la práctica poco explorado y donde la prevención es un aspecto fundamental para enfrentar el consumo de alcohol.

\section{Conclusiones}

Respondiendo al objetivo central de esta investigación que fue identificar el consumo de alcohol en mujeres universitarias del sur del estado de Veracruz en México; el estudio aporta datos importantes en cuanto al comportamiento del consumo de alcohol femenino; revelando que, si bien predominó el patrón de bajo riesgo, hay jóvenes que ingieren alcohol con patrones de consumo riesgoso y dependiente. Por ello, estos resultados pueden ser una base descriptiva entorno al fenómeno del consumo de alcohol particularmente en las mujeres, que estimule nuevas investigaciones que profundicen en el patrón de consumo, así como intervenciones de enfermería encaminadas a la promoción y prevención del consumo de alcohol.

\section{Limitaciones del estudio}

Algunas de las limitaciones del estudio pudieron haber sido el tamaño de la muestra, ya que solo se incluyó a estudiantes de una universidad, así como el haber realizado solo pruebas descriptivas, sin embargo, el artículo se encamino solo a una aproximación al fenómeno de estudio.

\section{Referencias}

1. Organización Panamericana de la Salud. Informe sobre la situación mundial del alcohol y la salud 2018 [Internet]. Washington: Organización Panamericana de la Salud; 2019 [citado 2020 nov 25]. Disponible en: https://iris.p aho.org/bitstream/handle/10665.2/51352/OPSNMH19012_spa.pdf?sequence=1\&isAllowed =y

2 Organización Mundial de la Salud. Alcohol [Internet]. Ginebra: Organización Mundial de la Salud; 2018 [citado 2019 sep. 05]. Disponible en: https://www.who.int/es/newsroom/factsheets/detail/alcohol

3 Organización Panamericana de la Salud. Alcohol [Internet]. Washington: Organización Panamericana de la Salud; 2020 [citado 2020 sep. 25]. Disponible en: https://www.paho.org/es/temas/alcohol

4 García C, Calvo F, Carbonell X, Giralt C. Consumo intensivo de alcohol y conductas sexuales de riesgo en población universitaria. Salud y Drogas [Internet]. 2017;17(1):63-71. Disponible en: http://www.redalyc.org/articulo.oa ?id $=83949782007$

5 Organización Panamericana de la Salud. Sinopsis de política: políticas tributarias y de precios del alcohol en la región de las américas [Internet]. Washington: Organización Panamericana de la Salud; 2019 [citado 2020 sep. 25]. Disponible en: https://iris.paho.org/bitstream/handle/10665.2/51529/opsnmh19020_spa.pdf?sequence=5\& isAllowed $=\mathrm{y}$

6 Cáceres D, Cáceres C, Colimil F, Ramírez D. Análisis interdisciplinar con perspectiva de género del alcoholismo en mujeres. Rev. Cuba. de Medicina Gen. Integral [Internet]. 2020;36(1):1-13. Disponible en: http://scielo.sld.cu /pdf/mgi/v36n1/1561-3038-mgi-36-01-e1056.pdf

7 Moncaleano JD, Brands B. Percepción de riesgo y conducción bajo los efectos del alcohol y la marihuana en estudiantes universitarios en un estudio multicéntrico: Colombia. Texto Contexto Enferm [Internet]. 2019;28:1-15. http://doi.org/10.1590/1980-265X-TCE-CICAD-24-28

8 Díaz M, Vargas D. Consumo de álcool entre estudantes de enfermagem. R de Pesq: cuidado é fundamental. 2017;9:64-70. http://doi.org/10.9789/2175-5361.2017.v9i1.64-70 
9 Secretaría de Salud. Encuesta Nacional de consumo de drogas, alcohol y tabaco (Encodat) 2016-2017 [Internet]. Bogotá: Secretaría de Salud; 2017 [citado 2019 ago. 03]. Disponible en: https://drive.google.com/file/d/1Iktp tvdu2nsrSpMBMT4FdqBIk8gikz7q/view

10 Instituto Nacional de Estadística y Geografía, Instituto Nacional de Salud Pública. Encuesta Nacional de Salud y Nutrición (Ensanut) 2018 [Internet]. Ciudad de México: Instituto Nacional de Estadística y Geografía; 2018 [citado 2020 sep. 26]. Disponible en: https://ensanut.insp.mx/encuestas/ensanut2018/informes.php

11 Alonso MM, Un LT, Armendáriz NA, Navarro EI, López MA. Sentido de coherencia y consumo de alcohol en jóvenes universitarios. Rev Invest Cienc Univ Autón Aguascalientes. 2018;26(75):66-72. Disponible en: https: //dialnet.unirioja.es/servlet/articulo?codigo $=6579487$

12 López JA, Morales FA. Consumo de tabaco y alcohol en estudiantes universitarios de enfermería de una institución pública del estado de Puebla, México. RFSC. 2018;5(1):8-13. http://doi.org/10.20320/rfcsudes.v5i1.101

13 Universidad de Alcalá. Alcohol deshidrogenasa [Internet]; 2018 [citado 2020 sep. 26]. Disponible en: http://ww w3.uah.es/chemevol/index.php/alcohol-deshidrogenasa/

14 Organización Mundial de la Salud. Audit [Internet]; 2020 [citado 2020 sep. 26]. Disponible en: https://apps.wh o.int/iris/handle/10665/67205

15 Secretaría General de los Estados Unidos Mexicanos. Reglamento de la Ley General de Salud en Materia de Investigación para la salud Última Reforma DOF 02-04-2014 [Internet]; 2014 [citado 2020 sep. 25]. Disponible en: http://www.diputados.gob.mx/LeyesBiblio/regley/Reg_LGS_MIS.pdf

16 Telumbre-Terrero JY, Esparza-Almanza SE, Alonso-Castillo BA, Alonso-Castillo MTJ. Actitudes hacia el consumo de alcohol y consumo de alcohol en estudiantes de enfermería. Investig Enferm Imagen Desarr [Internet]. 2017;19(2):69-81. http://doi.org/10.11144/Javeriana.ie19-2.ahca

17 Gómez Z, Landeros P, Pérez M, Martínez P. Consumo de alcohol, tabaco y otras drogas en jóvenes universitarios. Revista de Salud Pública y Nutrición [Internet]. 2017;16(4):1-9. Disponible en: http://www.redalyc.org/artic ulo.oa?id=79920065007

18 Garcés-Pérez GO, Zambrano-Constanzo AX. Significados en torno al desarrollo del consumo problemático y dependencia alcohólica en comunidades mapuches rurales de la región de la Araucanía, Chile, 2016-2017. Salud Colectiva [Internet]. 2019;15:1-18. http://doi.org/10.18294/sc.2019.1932

\section{Notas}

* Artículo original de investigación

\section{Licencia Creative Commons CC BY 4.0}

Cómo citar este artículo: Cortaza-Ramírez L, Blanco-Enríquez FE. Consumo de alcohol en mujeres universitarias del Sur del Estado de Veracruz, México. Investig Enferm Imagen Desarr. 2020;22. https://do i.org/10.11144/Javeriana.ie22.camu 